\title{
THE CAVALIERI ESTIMATOR WITH UNEQUAL SECTION SPACING REVISITED
}

\author{
MARKUS KIDERLEN ${ }^{\bowtie, 1}$ AND KARL-ANTON DORPH-PETERSEN ${ }^{2,3,4}$
}

${ }^{1}$ Centre for Stochastic Geometry and Advanced Bioimaging, Department of Mathematics, Aarhus University, Aarhus, Denmark; ${ }^{2}$ Centre for Stochastic Geometry and Advanced Bioimaging, Aarhus University, Aarhus, Denmark; ${ }^{3}$ Translational Neuropsychiatry Unit, Department of Clinical Medicine, Aarhus University, Aarhus, Denmark; ${ }^{4}$ Translational Neuroscience Program, Department of Psychiatry, University of Pittsburgh, Pittsburgh, PA, USA.

e-mail: kiderlen@math.au.dk, karl-anton@dorph-petersen.dk

(Received March 31, 2017; revised May 7, 2017; accepted May 7, 2017)

\begin{abstract}
The Cavalieri method allows to estimate the volume of a compact object from area measurements in equidistant parallel planar sections. However, the spacing and thickness of sections can be quite irregular in applications. Recent publications have thus focused on the effect of random variability in section spacing, showing that the classical Cavalieri estimator is still unbiased when the stack of parallel planes is stationary, but that the existing variance approximations must be adjusted. The present paper considers the special situation, where the distances between consecutive section planes can be measured and thus where Cavalieri's estimator can be replaced by a quadrature rule with randomized sampling points. We show that, under mild conditions, the trapezoid rule and Simpson's rule lead to unbiased volume estimators and give simulation results that indicate that a considerable variance reduction compared to the generalized Cavalieri estimator can be achieved.
\end{abstract}

Keywords: Cavalieri estimator, perturbed spacing, randomized Newton-Cotes quadrature, variance approximations.

\section{INTRODUCTION}

The Cavalieri estimator for the volume of a compact object $Y \subset \mathbb{R}^{n}$ is given by

$$
\hat{V}=h \sum_{i \in \mathbb{Z}} f(U+i h) .
$$

Here, for a fixed unit vector $u \in \mathbb{R}^{n}$, the measurement function

$$
f(x)=\lambda_{n-1}\left(Y \cap H_{x}\right)
$$

is the $(n-1)$-dimensional Lebesgue measure $\lambda_{n-1}$ of $Y$ intersected with the hyperplane $H_{x}=\left\{z \in \mathbb{R}^{n}: z \cdot u=\right.$ $x\}$. Furthermore, $h$ is a fixed positive constant and $U$ is a uniform random variable in the interval $[0, h]$. Hence, $\hat{V}$ is based on intersections of $Y$ with a stack of parallel hyperplanes at distance $h$ apart. It is not difficult to show that $\hat{V}$ is an unbiased estimator of the volume

$$
V=\int_{\mathbb{R}} f \mathrm{~d} x
$$

of $Y$. In applications, one usually considers the cases $n=2$ or $n=3$, but we work with the slightly more general setting of arbitrary $n \geq 1$. Also, the considerations below do actually not depend on the interpretation of $f(x)$ as the $(n-1)$-dimensional section volume, but only require that $f: \mathbb{R} \rightarrow \mathbb{R}$ is some
Lebesgue-integrable function with compact support. Under this assumption on $f$ the estimator in Eq. 1 is unbiased for $V$ given by Eq. 3. Classical theory (see (Baddeley \& Jensen, 2005, Section 13.2) and the references therein) yields variance approximations for $\hat{V}$ based on a general form of the Euler-Maclaurin formula for the geometric covariogram of $f$.

It was remarked in Baddeley et al. (2006) that the assumption of equidistant section spacing may not be satisfied in applications, for instance when $\hat{V}$ is derived from physical sections of a material. Hence, instead of at the ideal sampling positions $\{U+i h: i \in \mathbb{Z}\}, f$ is evaluated at the points in $X=$ $\left\{\ldots, x_{-2}, x_{-1}, x_{0}, x_{1}, \ldots\right\} \subset \mathbb{R}$, where one can think of $x_{i}$ as being a blurred version of the exact point $U+i h$. We will assume throughout that the points of $X$ are given in ascending order, that is,

$$
\cdots<x_{i-2}<x_{i-1}<x_{i}<x_{i+1}<x_{i+2}<\cdots
$$

The (random) point set $X$ will be considered as a point process in $\mathbb{R}$, and we assume throughout that $X$ is stationary; see the next section for details. The generalized Cavalieri estimator based on the sampling points in $X$ is given by

$$
\hat{V}=h \sum_{x_{i} \in X} f\left(x_{i}\right)
$$



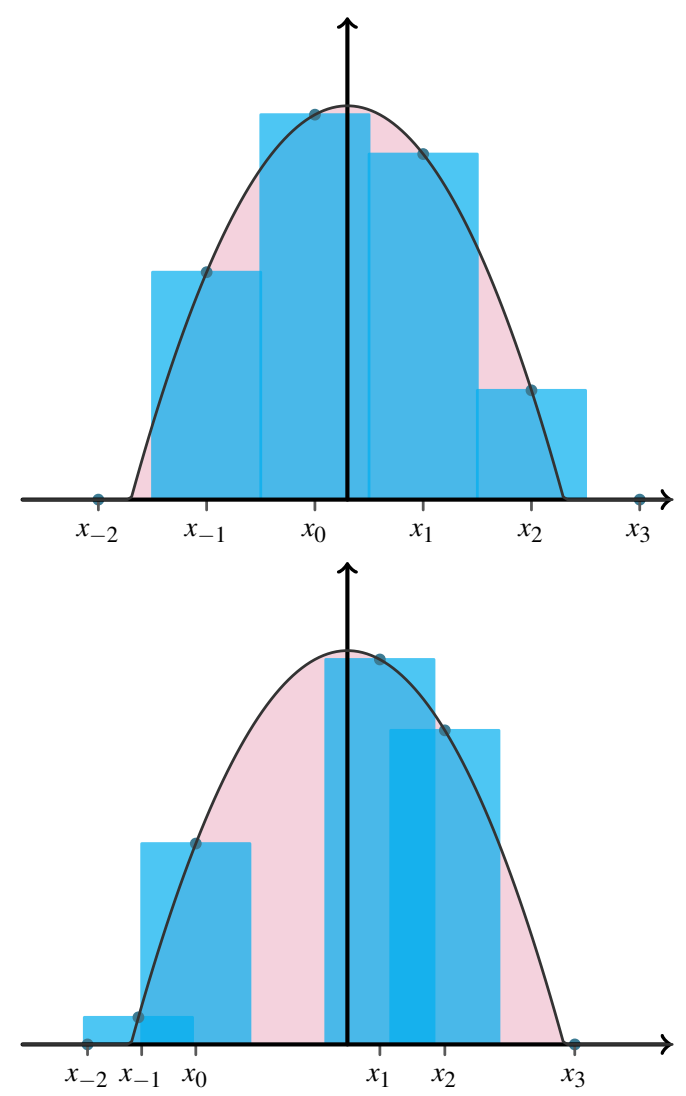

Fig. 1. The rectangular quadrature rule for equidistant points $\left(x_{i}\right)$ as used in the classical Cavalieri estimator (top) and the corresponding rule when applied to points that are not equidistant (bottom). The measurement function is in both cases $f(x)=2\left(1-x^{2}\right)$ for $x \in[-1,1]$.

with $h$ now being the average section spacing. It is still unbiased for $V$, but classical variance predictors may underestimate the true variance substantially; see Baddeley et al. (2006). In the two followup papers Ziegel et al. (2010) and Ziegel et al. (2011) the variances have been calculated under three realistic error models and variance predictors based on the observed values $f\left(x_{i}\right), x_{i} \in X$, are derived. In particular, the model with cumulative error, where the increments between successive sampling locations are independent and identically distributed, exhibits a rather drastic variance increase compared to the equidistant case. A geometric comparison between the classical Cavalieri estimator in the equidistant case and the generalized Cavalieri estimator in the perturbed case, is shown in Fig. 1.

In the following we argue that this undesirable behavior can be avoided if more information on the positions of the sections can be collected. This is detailed in the following main section. In certain applications it is possible to study the collection of serial sections after the actual section process.

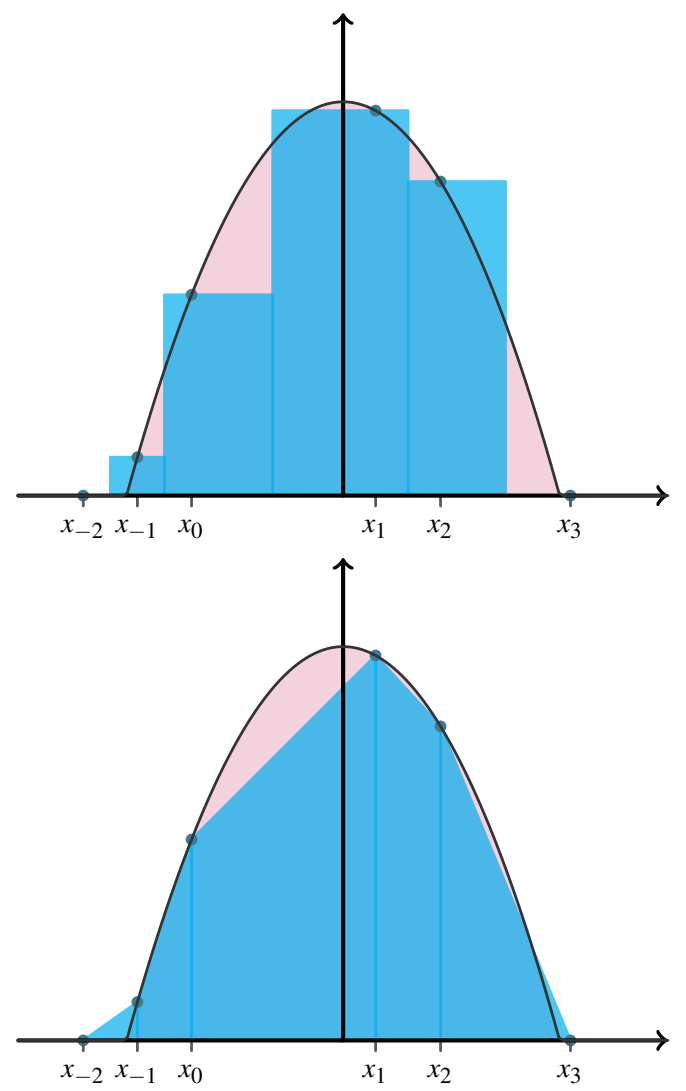

Fig. 2. The same measurement function and sample points as in the lower Fig. 1, but now the midpoint rule is used (top) leading to non-overlapping rectangles with varying base lengths. The trapezoid rule (bottom) approximates with a piecewise linear function and leads to the same estimator as the midpoint rule.

This is typically the case when sections originate from the surfaces of thick parallel slabs of uneven thickness. Thus, one example is when the individual slab thicknesses (corresponding to the individual intersectional distances) may be measured directly using a micrometer or digital caliper. Another example is when the final set of slabs may be observed and imaged from the side relative to the faces of the slabs; see Fig. 1 in Baddeley et al. (2006) for the kind of images that we have in mind. In the latter case, individual section positions may then be determined from the image.

The precise general assumptions for the present investigations are that we can

(i) keep track of the order of the sections,

(ii) (at least approximately) measure the distances between consecutive sections.

Note that many classical stereological estimators do not require this additional information, as they rely exclusively on samples with planar sections, but not 
on their relative positions. However, if this information is available, one can use quadrature formulae to obtain better approximations to the integral of the measurement function.

The first method that might come to mind is the midpoint rule, illustrated in Fig. 2 (top). Here, the integral of the measurement function $f$ between two sections planes at consecutive positions $x_{i}$ and $x_{i+1}$ is approximated by

$$
\frac{x_{i+1}-x_{i}}{2} f\left(x_{i}\right)+\frac{x_{i+1}-x_{i}}{2} f\left(x_{i+1}\right),
$$

and summing over all intervals would give the estimator

$$
\hat{V}=\sum_{x_{i} \in X} \frac{x_{i+1}-x_{i-1}}{2} f\left(x_{i}\right)
$$

Note that the sum in Eq. 6 is finite: we have $f\left(x_{i}\right)=0$ for all but finitely many $i \in \mathbb{Z}$ as $f$ has compact support. Using the trapezoid rule as in Fig. 2, would yield an approximation of the integral between $x_{i}$ and $x_{i+1}$ given by

$$
\frac{f\left(x_{i+1}\right)+f\left(x_{i}\right)}{2}\left(x_{i+1}-x_{i}\right),
$$

which coincides with Eq. 5. Hence, both rules lead to the same estimator given by Eq. 6 . The trapezoid rule corresponds to approximating $f$ by a piecewise linear function, with the important difference to classical quadrature that this approximation is based on random sampling points. Higher order NewtonCotes quadrature rules can be applied as well, in a composite way. This will be outlined in the next section. The main results of the subsequent section are Corollaries 2 and 3, which state that the trapezoid rule and Simpson's rule both yield unbiased estimators for the integral of the measurement function. For the trapezoid rule the only requirement is that the point process $X$ is stationary. For Simpson's rule, an integrability condition must be satisfied. We continue the technical report with a section on simulation examples illustrating the variance reduction achieved by the new estimators, and finish it with a short section on Conclusions and future work.

\section{QUADRATURE RULES AT RANDOM POINTS}

Throughout the following we assume that the set $X$ of sampling locations is a stationary point process on $\mathbb{R}$ with intensity $\gamma>0$. Then $h=1 / \gamma$ is the average section spacing. For background material on point processes we refer to Chapter 3 in the monograph Schneider \& Weil (2008). Recall that we assume $f: \mathbb{R} \rightarrow \mathbb{R}$ to be an integrable function with compact support. Newton-Cotes rules (see, e.g., Stoer $\&$ Bulirsch (1980)) for numerical integration of order $n \in \mathbb{N}_{0}$ on an interval $\left[x_{i}, x_{i+n}\right]$ approximate a function $f(x)$ that is only known at the points $x_{i}, \ldots, x_{i+n}$ by the unique interpolating polynomial $p_{n}(x)$ of degree at most $n$ through the points $\left(x_{j}, f\left(x_{j}\right)\right)_{j=i}^{i+n}$. Then $\int_{x_{i}}^{x_{i+n}} f(x) \mathrm{d} x$ is approximated by

$$
\int_{x_{i}}^{x_{i+n}} p_{n}(x) \mathrm{d} x .
$$

Commonly, the points $x_{i}, \ldots, x_{i+n}$ are equidistant, but we will need the more general form here. The polynomial $p_{n}(x)$ can be expressed in Lagrange form as

$$
p_{n}(x)=\sum_{j=i}^{i+n} L_{j}(x) f\left(x_{j}\right)
$$

with the Lagrange basis polynomials $L_{j}(x)=$ $\prod_{k \neq j} \frac{x-x_{k}}{x_{j}-x_{k}}$. Hence, the integral in Eq. 7 is of the form

$$
I_{i}=\sum_{j=i}^{i+n} \beta_{j}^{(i)} f\left(x_{j}\right)
$$

with $\beta_{j}^{(i)}=\int_{x_{i}}^{x_{i+n}} L_{j}(x) \mathrm{d} x$. A partial integration argument shows that $\beta_{j}^{(i)}$ can be expressed in terms of the increments $h_{k}=x_{k}-x_{k-1}, k=i+1, \ldots, i+n$.

Usually, this quadrature is only used for small values of $n$ to avoid Runge's phenomenon, that is, oscillations of high order interpolating polynomials destroying the quality of the approximation. Instead, the $n$th order approximation is applied to consecutive blocks of $n$ intervals, a method that is usually referred to as composite quadrature. In the present context, where $f$ is known at all the points $x_{i}$ of a point process, the sum $I_{i}$ in Eq. 8 can be calculated for any $i \in \mathbb{N}$, and a composite integral approximation with start $k \in \mathbb{Z}$ is

$$
I_{k}^{\text {comp }}=\sum_{i \in \mathbb{Z}} I_{k+i n} .
$$

Clearly, $I_{k}^{\text {comp }}=I_{k+n}^{\text {comp }}$, and there is no reason to prefer one start $k$ in $\{1, \ldots, n\}$ to any other, so we let the final quadrature rule be given as the mean of these $n$ numbers $\hat{V}_{n}=(1 / n) \sum_{k=1}^{n} I_{k}^{\text {comp }}$, when $n>0$. For $n=0$ we put $\hat{V}_{0}=I_{1}^{\text {comp }}$, an estimator that is obtained from the rectangular rule. In contrast to an equidistant setting, this rectangular rule is in general different from the midpoint rule. In view of Eq. 8 and Eq. 9 we have

$$
\hat{V}_{n}=\sum_{x \in X} \alpha_{x}(X) f(x)
$$


where the weights $\alpha_{x}(X) \in \mathbb{R}$ only depend on the $n$ increments to the left and the right of $x$. More precisely, if $X=\left(x_{i}\right)$ is ordered,

$$
\alpha_{x_{i}}(X)=\tilde{\alpha}\left(h_{i-n+1}, \ldots, h_{i}, \ldots, h_{i+n}\right),
$$

where $\tilde{\alpha}$ is some function of $2 n$ of the increments $h_{j}=x_{j}-x_{j-1}, j \in \mathbb{Z}$. We will call $n$ the order of the estimator in Eq. 10. In the next section, $\alpha$ will be determined for the quadrature of order $n=2$. Note that Eq. 11 implies that $\alpha$ is translation invariant, in the sense that

$$
\alpha_{x+z}(X+z)=\alpha_{x}(X)
$$

for all $x \in X$ and $z \in \mathbb{R}$.

\section{UNBIASEDNESS}

To discuss the unbiasedness of the estimator in Eq. 10, a measurability assumption for $\alpha$ is needed. In view of Eq. 12 we consider the Palm distribution $\mathbb{P}^{0}$ of $X$, that is, the conditional distribution of $X$ given that the origin is a point of $X$ and require that $\alpha_{0}(X)$ is quasi-integrable with respect to $\mathbb{P}^{0}$. Quasiintegrability means here that $\alpha_{0}(\cdot)$ is measurable and at least one of the numbers $\mathbb{E}^{0} \alpha_{0}^{+}(X)$ and $\mathbb{E}^{0} \alpha_{0}^{-}(X)$ is finite. Here and in the following we write $\mathbb{E}^{0}$ for the expectation with respect to the Palm distribution $\mathbb{P}^{0}$. When $X$ has a point at the origin, we will always apply the convention that the ordered sequence $X=\left(x_{i}\right)$ satisfies $x_{0}=0$. Standard facts on the Palm distribution of a stationary point process can be found in Sections 3.3 and 3.4 in Schneider \& Weil (2008). For the present investigations it is enough to note that the Palm distribution is invariant under bijective point shifts. In particular, it is unaltered if the point process $X$ is shifted in such a way that the origin is moved to its $k$ th next right neighbor in $X$; see, for instance, Satz 4.3 in Mecke (1975) or Theorem 3.1 in Heveling \& Last (2005). This implies that

$$
\mathbb{E}^{0} f\left(h_{1}, \ldots, h_{n}\right)=\mathbb{E}^{0} f\left(h_{k+1}, \ldots, h_{k+n}\right)
$$

for all measurable nonnegative functions $f$ of $n$ increments and all $n \in \mathbb{N}, k \in \mathbb{Z}$. The increment between the typical point $x_{0}=0$ and its next neighbor to the right has Palm expectation

$$
\mathbb{E}^{0} h_{1}=\frac{1}{\gamma}
$$

We can now state the first result, which does not rely on the fact that $\alpha$ is obtained from a Newton-Cotes quadrature.
Theorem 1. Let $X$ be a stationary point process with Palm distribution $\mathbb{P}^{0}$ and intensity $0<\gamma<\infty$. Assume $\alpha:(x, X) \mapsto \alpha_{x}(X)$ satisfies Eq. 12 and that $\alpha_{0}$ is quasi-integrable with respect to $\mathbb{P}^{0}$. Then

$$
\hat{V}=\sum_{x \in X} \alpha_{x}(X) f(x)
$$

is unbiased for $\gamma \mathbb{E}^{0}\left[\alpha_{0}(X)\right] \int_{\mathbb{R}} f(x) \mathrm{d} x$.

Proof. This follows from the refined Campbell theorem (Theorem 3.3.3 in Schneider \& Weil (2008)) by the invariance properties of $\alpha$ and the stationarity of $X$.

The simplest instance of $\hat{V}$ is the one where $\alpha$ is a constant. If we put $\alpha \equiv 1 / \gamma$, Theorem 1 shows that $\hat{V}=\frac{1}{\gamma} \sum_{x \in X} f(x)$ is unbiased for $\int_{\mathbb{R}} f(x) \mathrm{d} x$. This result also follows from Theorem 1 in Baddeley et al. (2006), and was the basis for their investigation. The classical Cavalieri estimator is a special case of this setting and is obtained when the points of $X$ are equidistant.

Example 1. Applying the trapezoid rule we obtained the estimator in Eq. 6, which coincides with Eq. 15 when $\alpha_{x_{i}}(X)=\left(h_{i}+h_{i+1}\right) / 2$. By Eq. 13 and Eq. 14 we have

$$
\mathbb{E}^{0} \alpha_{0}(X)=\mathbb{E}^{0} \frac{h_{0}+h_{1}}{2}=\frac{1}{\gamma}
$$

Hence, $\hat{V}_{1}$ given by the trapezoid rule is unbiased for $\int_{\mathbb{R}} f(x) \mathrm{d} x$.

Corollary 2. The estimator $\hat{V}_{1}$ based on the trapezoid rule (or, equivalently, on the midpoint rule) and given by Eq. 6 is unbiased for $\int_{\mathbb{R}} f(x) \mathrm{d} x$. Furthermore $\hat{V}_{1} \geq 0$ when $f \geq 0$.

The last property is useful in a Cavalieri setting, where the measurement function $f(x)$ is the area of the hyperplane section with offset $x$ from the origin, and thus nonnegative. Then, the volume estimate $\hat{V}_{1}$ is nonnegative, as well. This monotonicity need not hold for higher order quadrature rules.

Example 2. Simpson's rule.

Simpson's rule uses polynomial approximation of order $n=2$. An easy calculation (see Selmer (1958)) shows that $I_{i}$ satisfies Eq. 8 with

$$
\begin{aligned}
& \beta_{i}^{(i)}=\frac{h_{i+1}+h_{i+2}}{6} \frac{2 h_{i+1}-h_{i+2}}{h_{i+1}}, \\
& \beta_{i+1}^{(i)}=\frac{\left(h_{i+1}+h_{i+2}\right)^{3}}{6 h_{i+1} h_{i+2}}, \\
& \beta_{i+2}^{(i)}=\frac{h_{i+1}+h_{i+2}}{6} \frac{2 h_{i+2}-h_{i+1}}{h_{i+2}} .
\end{aligned}
$$


Thus,

$$
\hat{V}_{2}=\sum_{x \in X} \alpha_{x}(X) f(x)
$$

with

$$
\begin{aligned}
\alpha_{x_{i}}(X)= & \frac{1}{2}\left(\beta_{i}^{(i-2)}+\beta_{i}^{(i-1)}+\beta_{i}^{(i)}\right)=\frac{1}{12 h_{i} h_{i+1}} \\
\times & \left(h_{i-1} h_{i} h_{i+1}+h_{i} h_{i+1} h_{i+2}+h_{i}^{3}+h_{i+1}^{3}+\right. \\
& \left.5 h_{i}^{2} h_{i+1}+5 h_{i} h_{i+1}^{2}-h_{i-1}^{2} h_{i+1}-h_{i} h_{i+2}^{2}\right) .
\end{aligned}
$$

Hence, by Eq. 13 and Eq. 14,

$$
\begin{aligned}
\mathbb{E}^{0} \alpha_{0}(X)=\frac{1}{\gamma} & +\frac{1}{12} \mathbb{E}^{0}\left(\frac{h_{i}^{2}}{h_{i+1}}-\frac{h_{i-1}^{2}}{h_{i}}\right) \\
& +\frac{1}{12} \mathbb{E}^{0}\left(\frac{h_{i+1}^{2}}{h_{i}}-\frac{h_{i+2}^{2}}{h_{i+1}}\right)=\frac{1}{\gamma},
\end{aligned}
$$

if

$$
\mathbb{E}^{0} \frac{h_{1}^{2}}{h_{0}}<\infty \quad \text { and } \quad \mathbb{E}^{0} \frac{h_{0}^{2}}{h_{1}}<\infty .
$$

In the particular case where all the points of the stationary point process $X$ are a.s. equidistant, $\alpha_{x_{i}}(X)=h_{1}$ is equal to the increment between two consecutive points, and Simpson's rule coincides with the trapezoid rule.

We combine the findings in the last example with Theorem 1 to obtain the following Corollary.

Corollary 3. Assume that the integrability conditions in Eq. 18 hold. Then, the estimator $\hat{V}_{2}$ based on Simpson's rule, given by Eq. 16 and Eq. 17, is unbiased for $\int_{\mathbb{R}} f(x) \mathrm{d} x$.

In applications, the increments are usually bounded and Eq. 18 can then be replaced by the assumption $\mathbb{E}^{0} h_{1}^{-1}<\infty$.

It is straightforward to construct examples of stationary point processes $X$ and non-negative measurement functions $f$ such that $\hat{V}_{2}<0$ with positive probability. However, in our simulations with realistic choices of a model for $X$ and natural measurement functions, we never encountered a negative realization of this estimator. This indicates that the problem of a negative $\hat{V}_{2}$ is not a severe one in practical applications.

\section{IMPROVED VARIANCE}

For exactly equidistant sampling points, the variance of the classical Cavalieri estimator $\hat{V}$ in Eq. 1 is commonly decomposed as

$$
\operatorname{var}(\hat{V})=\operatorname{var}_{E}(\hat{V})+Z(h)+r(h),
$$

where the extension term $\operatorname{var}_{E}(\hat{V})$ describes the dominating overall trend of the variance, $Z(t)$ is an oscillating term, the so-called Zitterbewegung, and $r(h)$ is a lower order remainder. Usually, the extension term is of the form

$$
\operatorname{var}_{E}(\hat{V})=c \cdot h^{\alpha},
$$

with an exponent $\alpha>0$, which depends only on smoothness properties of the measurement function $f$, and some positive constant $c$; see p. 307 in Baddeley $\&$ Jensen (2005). For instance, if the volume of the unit ball $B^{3}$ in $\mathbb{R}^{3}$ is estimated from sections with twodimensional planes, the measurement function satisfies

$$
f_{B^{3}}(x)=\pi\left(1-x^{2}\right), \quad-1 \leq x \leq 1 .
$$

The trivial extension of this function on $\mathbb{R}$ is $(m, \infty)$ piecewise smooth with $m=1$, meaning that the derivatives up to order $m-1$ exist and are continuous, whereas all higher order derivatives have a finite number of jumps of finite size. For the classical Cavalieri estimator with equidistant sampling points and measurement function given by Eq. 20, Eq. 19 holds with $c=\pi^{2} / 90$ and $\alpha=2 m+2=4$.

The purpose of this section is to present Monte Carlo simulations supporting the claim that the above defined quadrature rules decrease the variance of the estimator compared to the generalized Cavalieri estimator when the spacing is perturbed. We adopt a perturbation model similar to the one in Ziegel et al. (2011) where we generated a stationary point process $\{U+i h: i \in \mathbb{Z}\}$ of ideally equidistant points (and $U$ uniform in the interval $[0, h]$ ). The perturbed sampling points $x_{i}=U+i h+D_{i}$ where then obtained by adding stochastically independent and identically distributed random errors $\left(D_{i}\right)$, which were also independent of $U$. The distribution of $D_{i}$ was uniform in the interval $[-s h / 2, s h / 2]$, where $s$ was chosen in such a way that the average relative deviation (i.e. the coefficient of variation) of $h+D_{i}$ from the ideal increment $h$ was $5 \%$. Fig. 3 shows the empirical variances of three estimators from 2000 Monte Carlo simulations as a function of the average number of sections, that is, $2 \gamma$ with $\gamma=1 / h$. The measurement function in Eq. 20 for the estimation of the volume of the three-dimensional unit ball was used. The variances of the two new estimators $\hat{V}_{1}$ and $\hat{V}_{2}$ are virtually identical, and their 
overall trend appears to coincide with the extension term of the classical Cavalieri estimator given in Eq. 19 with $\alpha=4$, indicated in black in the figure. Fitting a least squares line to the variances of the two new estimators yields an approximate exponent $\hat{\alpha}=3.97$ for the extension term, strongly indicating, that the asymptotic behavior is comparable to the classical Cavalieri estimator. In contrast to this, the empirical variance of the generalized Cavalieri estimator is always larger, and has an theoretical exponent of $\alpha=3$ only.

It is amazing that piecewise linear approximation in $\hat{V}_{1}$ and piecewise quadratic approximation in $\hat{V}_{2}$ lead to the same variance behavior. The reason for this appears to be the fact that the measurement function in Eq. 20 is not continuously differentiable at the endpoints of its support. We therefore repeated the investigations with a spindle shaped body $S$ with peaked ends as illustrated in Fig. 4. Its measurement function is

$$
f_{S}(x)=\frac{\pi}{2}(1+\cos \pi x), \quad-1 \leq x \leq 1 .
$$

This function is $(2, \infty)$-piecewise smooth. For the classical Cavalieri estimator with equidistant sampling points Eq. 19 thus holds with $c=\pi^{6} / 60480$ and $\alpha=$ $2 m+2=6$.

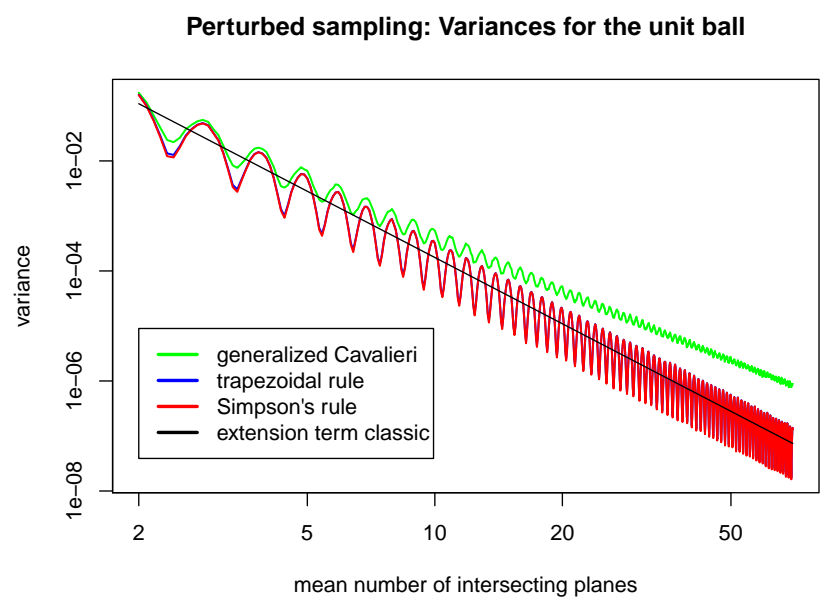

Fig. 3. The empirical variance for volume estimation of the unit ball $B^{3}$ in $\mathbb{R}^{3}$, shown in a double logarithmic plot. The $x$-coordinate is $2 \gamma$ and the black line is the extension term in Eq. 19 for the classical Cavalieri estimator with section spacing $1 / \gamma$.

Fig. 5 shows the empirical variances for the volume estimation for $S$. Again, the variance of the generalized Cavalieri estimator follows a line with approximate slope $-\alpha=-3$. This is in accord with the theoretical result in Ziegel et al. (2010) implying that this exponent holds for all $(m, \infty)$ piecewise smooth $f$ if $m \geq 1$. In contrast to this, the estimates based on trapezoid or Simpson's rule have approximately the same slope as the extension term in the equidistant case, that is, -6 . The smoothness of the measurement function can apparently be better exploited by Simpson's rule: the corresponding variance is never larger than $\operatorname{var}\left(\hat{V}_{1}\right)$. The double logarithmic scale makes it difficult to see the precise gain, but to give an example: For $\gamma=5$ the empirical standard deviation of $\hat{V}_{1}$ is about twice as large as the one of $\hat{V}_{2}$. Numerical simulations with even smoother measurement functions result in variance behavior, where the empirical exponent $\alpha$ is strictly larger for $\operatorname{var}\left(\hat{V}_{2}\right)$ than for $\operatorname{var}\left(\hat{V}_{1}\right)$.

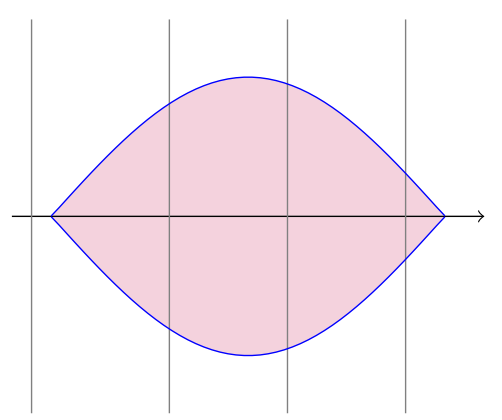

Fig. 4. Planar section of a three-dimensional body $S$ of revolution through its horizontal rotational axis. The boundary of this spindle is determined by the functions $u_{ \pm}(x)= \pm \sqrt{(1+\cos \pi x) / 2},-1 \leq x \leq 1$. The section planes (indicted as vertical lines) are all orthogonal to the rotational axis.

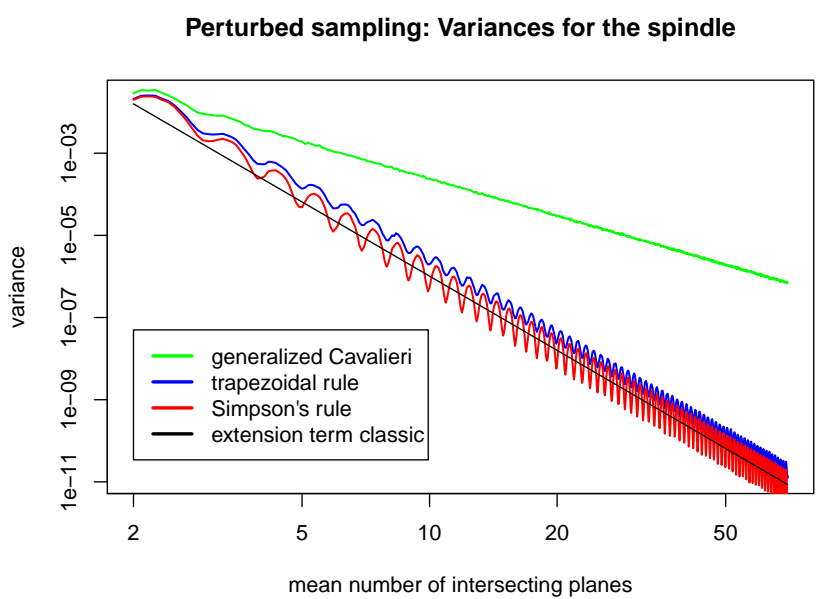

Fig. 5. The empirical variances for volume estimation of the spindle $S$ shown in a double logarithmic plot. The $x$-coordinate is $2 \gamma$ and the black line is the extension term in Eq. 19 for the classical Cavalieri estimator with section spacing $1 / \gamma$. 


\section{CONCLUSIONS AND FUTURE WORK}

We have shown that estimators based on classical quadrature rules are good alternatives for the generalized Cavalieri estimator when distances between consecutive sampling points vary and are known. The estimator based on the trapezoid rule is unbiased as long as the underlying point process of sampling points is stationary, which means roughly speaking that no part of $\mathbb{R}$ is sampled differently from any other part of $\mathbb{R}$. The estimator that uses Simpson's rule requires in addition a mild integrability condition to guarantee unbiasedness. Simulations showed that variances are reduced when the sampling points are independently and identically perturbed. Apparently, the variance reduction is largest when the measurement functions are smooth, as the generalized Cavalieri estimator cannot take advantage of the additional smoothness. This effect is expectedly even stronger, when a cumulative error model ("meat slicer model") for the sampling points is employed. When the measurement function is $(m, \infty)$-piecewise smooth with $m \geq 2$, the trapezoid rule and Simpson's rule yield different variance behavior. In many stereological applications such a smoothness cannot be guaranteed, which means that the trapezoid rule should be used. However, in other applications of systematic geometric sampling smoothness of $f$ may be more natural and a higher order quadrature rule thus profitable.

Concerning practical applications, the following recommendations may be helpful.

1. The classical Cavalieri estimator is typically easy to implement in real-world applications and is thus the natural starting point for a stereological investigation. If one suspects that non-equidistant sampling positions might be a severe issue, the section protocol should be planned in advance in such a way that approximative sampling distances can be determined later.

2. If subsequently it is discovered that the application is burdened by non-equidistant sampling, the Cavalieri estimator is still unbiased but the variance is more or less inflated, which can be assessed using the previously published variance approximations.

3. If it is also possible to obtain the exact positions of the (non-equidistant) sampling sites, the above defined alternative estimators can be used. They are unbiased and appear to match the variance of the classical Cavalieri estimator (the latter being applied to exactly equidistant sample points). We expect that the use of the trapezoid rule is preferable, as it is easier to implement than higher order quadratures and does not require any integrability condition for unbiasedness.

In future work we intend to find explicit formulae for the variance depending on the model of $X$, similar to Theorem 1 in Baddeley et al. (2006). However, it will no longer be sufficient to know the second order properties of $X$, calling for the use of more advanced tools from point process theory. It is an interesting question if reasonable upper bounds for $\operatorname{var}\left(\hat{V}_{n}\right)$ can still be established under realistic assumptions on $X$. If so, the next step would be a covariogram model allowing for the prediction of the variance solely from the observed data. In addition, it is important to determine the robustness of the new estimators in situations were the positions of the non-equidistant sampling sites are only approximately known, as the exact positions may be unobtainable in practice. Finally, it might be profitable to compare the above estimators $\hat{V}_{n}$ with estimators that are based on quadrature rules using other approximating functions, such as splines.

\section{ACKNOWLEDGEMENTS}

This research was supported by Centre for Stochastic Geometry and Advanced Bioimaging, funded by a grant from the Villum Foundation.

\section{REFERENCES}

Baddeley A, Dorph-Petersen KA, Jensen EBV (2006). A note on the stereological implications of irregular spacing of sections. J Microsc 222:177-81.

Baddeley A, Jensen EBV (2005). Stereology for Statisticians. New York: Chapman \& Hall.

Heveling M, Last G (2005). Characterization of Palm measures via bijective point shifts. Ann Probab 33:1698-715.

Mecke J (1975). Invarianzeigenschaften allgemeiner Palmscher Maße. Math Nachr 65:335-44.

Schneider R, Weil W (2008). Stochastic and Integral Geometry. New York: Springer.

Selmer ES (1958). Numerical integration by non-equidistant ordinates. Nord Matem Tidskr 6:97-108.

Stoer J, Bulirsch R (1980). Introduction to Numerical Analysis. New York: Springer.

Ziegel J, Baddeley A, Dorph-Petersen KA, Jensen EBV (2010). Systematic sampling with errors in sample locations. Biometrika 97:1-13.

Ziegel J, Jensen EBV, Dorph-Petersen KA (2011). Variance estimation for generalized Cavalieri estimators. Biometrika 98:187-98. 